\title{
Foreword-cognitive radio: from equipment to networks
}

\author{
Christophe Moy $\cdot$ Linda Doyle $\cdot$ Yukitoshi Sanada
}

(C) Institut TELECOM and Springer-Verlag 2009

\section{Introduction}

Cognitive radio is a technology that is of growing interest internationally. Currently, there is a highly active worldwide community of academic and industrial researchers working in this field. Efforts focus on the many technical questions as well as the economic and political challenges of moving this new technology from research concept to reality $[1,2]$. Cognitive radio holds much promise not just in the military domain but increasingly in the civil and commercial domain.

The boom of wireless commercial markets started in the early 1990s and has resulted in an ever-increasing demand for wireless connectivity and greater capacity to facilitate the variety of data applications in daily use. In the world of mobile communications, UMTS/3G networks are part of the response to that demand. The birth of $3 \mathrm{G}$ services served to highlight the demand for spectrum with recordbreaking prices paid for the $3 \mathrm{G}$ bands in many countries in Europe. The management of $3 \mathrm{G}$ spectrum and indeed management of spectrum in general has lead to much discussion and debate - in general, there is a strong belief

\footnotetext{
C. Moy $(\bowtie)$

SUPELEC/IETR,

Rennes, France

e-mail: christophe.moy@supelec.fr

L. Doyle

Trinity College,

Dublin, Ireland

e-mail: ledoyle@tcd.ie

Y. Sanada

Keio University,

Tokyo, Japan

e-mail: sanada@elec.keio.ac.jp
}

that the perceived scarcity of spectrum is very much related to how it is managed. Crucially in this debate, cognitive radio is seen as a technology that can facilitate new and more dynamic ways of providing access to spectrum and addressing the scarcity problem.

It is time to reconsider the century-old paradigm of fixed dedicated frequency allocations. But it is hard to change habits of tens of years. However, we already see evidence of change. The Federal Communications Commission (FCC) in the USA has been working since 2001 on software-defined radio [3] in which the operating parameters of frequency range, modulation type, or maximum output power (either radiated or conducted) can be altered by making a change in software that controls the operation of the device without making any changes in the hardware components that affect the radio frequency emissions. In 2002, the FCC maintained that authorizing more flexible spectrum uses promises both immediate and future innovations without consuming additional spectrum resources [4], opening the door to cognitive radio. On 4 November 2008, the FCC voted 5-0 to approve the unlicensed use of white space (there are five FCC commissioners). This was followed by the release of what is known as a Second Report and Order, ETDocket no. 04-186 and ET Docket no. 02-380, which detail the rules under which unlicensed devices could use the TV white spaces. The rules provide for the operation of both fixed and portable unlicensed devices in the TV bands. As cognitive radio is seen as a key player in the use of TV white space, this has served to further open the door for cognitive radio. Even in Japan where spectrum is fully controlled by the government, there is a large demand to move to a more dynamic spectrum management and national research projects regarding the cognitive radio technology is ongoing [5]. In the UK, the regulator Ofcom has also made encouraging remarks. In fact, as December 2007, Ofcom proposed to 
allow cognitive radio to use what they call "interleaved spectrum" provided that it can be shown that this does not cause interference to other spectrum users.

On the standards front, there is also much activity. Spectrum sharing concepts are integrated in the thinking of several Institute of Electrical and Electronics Engineers (IEEE) standards elaboration. For example, IEEE 802.22 is aiming to create wireless regional area networks based on constraint-based routing through the secondary usage of TV bands. IEEE SCC41 efforts are also developing standards for technologies, architectures, and facilitators to realize dynamic spectrum access networks. Furthermore, IEEE 802.16h and IEEE 802.11y are working on interference management and efficient resource allocation in shared (e.g., unlicensed) bands. At the European level, ETSI reconfigurable radio systems is exploring dynamic spectrum usage and joint radio resource management for multi-standard radio access as well as cognitive pilot channel solution.

What is needed to take cognitive radio from research concept to commercial reality? It is clear that part of the answer is an enabling regulatory environment, signs of which we are beginning to see. Though much more is needed. Cognitive radio is not an evolution. It is a revolution. This may frighten many wireless stakeholders. Though the business models are not yet clear, cognitive radio can be a source of profit for innovative and adaptive companies. In the meantime, the continuation of the scientific and technology research that underpins the field is vital to maintain the momentum and derive new ideas that could convince and comfort all concerned stakeholders. This special issue on cognitive radio is a brick in the wall of this building. As suggested by the subtitle, both cognitive radio equipment and cognitive radio networks are considered in this issue. On the one hand, network level studies and analyses have the ability to show the benefit of cognitive radio communication systems and quantify their impact. On the other hand, equipment design perspectives serve to improve the enabling technologies, including the all-important cognitive radio terminals, which are vital for the functioning of any future cognitive networks.

\section{This special issue}

There are nine papers in this special issue. The special issue begins with a paper from Joseph Mitola "Cognitive radio architecture evolution." This presents an overview of dynamic spectrum access and then goes on to show how cognitive radio architectures can evolve towards a "quality of experience." Mitola introduces a new concept of quality of information. He uses an interdisciplinary perspective and states that this requires advances in policy language architectures. He proposes cognitive linguistics for such languages in the paper. Their opening paper is followed by two papers on cognitive radio equipment. In the first one, "A generic cognitive framework for supervising the radio dynamic reconfiguration. An AI approach based on design problem classification," Nicolas Colson, Apostolos Kountouris, Armelle Wautier, and Lionel Husson explore cognitive mechanisms that should be embedded in cognitive radio equipment in order to achieve the required intelligent behavior. They present a solution for autonomous decision making based on multiple operational objectives. This is based on a mixing of learning and reasoning processes. In the second paper entitled "An executable metamodel of a hierarchical and distributed architecture management for cognitive radio equipments," Loïg Godard, Christophe Moy, and Jacques Palicot propose a design framework to integrate all necessary elements to manage the cognitive features of a cognitive radio, e.g., sensing, decision making, and reconfiguration. They derive a cognitive management architecture and have built a metamodel of this architecture as well as a simulator that enables the specification and test of equipment at a high level of abstraction while playing cognitive radio scenarios. Then, the special issue turns to cognitive networks. The paper from Panagiotis Demestichas "Enhanced network selections in a cognitive wireless B3G world" addresses the issue of directing terminals at the most appropriate radio networks so that it ensures optimal performance. The proposed solution has the ability to learn the quality of service capabilities of candidate networks at a low computational effort and a higher reliability. Then, Christian Doerr, Dirk Grunwald, and Douglas C. Siker, in their article "Local control of cognitive radio networks," take inspiration from swarm behavior as found in schools of fish or flocks of birds for the local control of cognitive radio networks. They propose swarm intelligence as a good means of efficient coordination of cognitive radio networks using local information only. In the sixth paper "Traffic modeling and forecasting using genetic algorithms for next-generation cognitive radio applications," Duminda Thilakawardana and Klaus Moessner explore the possibilities of forecasting network traffic using genetic algorithms. The goal is to manage and optimize the network load and the radio resources. In addition, the model also offers great benefit in a cognitive environment with its intrinsic learning capabilities. Then, the paper by Behrouz Maham, Are Hjorungnes, and Mérouane Debbah, "Power allocations in minimum-energy SER-constrained cooperative networks," focuses on power allocation strategies for the multi-branch, multi-hop networks. Several amplify-and-forward cooperative schemes are proposed, taking into account the statistical channel state information, the residual battery energy, and the quality-of-service requirements. In the eighth paper, "An optimal OSA approach based on 
channel-usage estimate under collision probability constraint in cognitive radio systems," Qinghai Xiao, Yunzhou Li, Ming Zhao, Shidong Zhou, and Jing Wang propose an opportunistic spectrum access approach based on a channel usage pattern in order to bound the collision probability when secondary users attempt to reach the spectrum holes. Finally, the last paper by T. Charles Clancy, "Dynamic spectrum access using the interference temperature model," deals with the use of an interference temperature model for controlling spectrum access. The article analyzes the viability of this model and proposes a medium access protocol and physical layer implementation.

With this special issue, the guest editors hope that the readers can use the research presented to develop new solutions. They thank all the anonymous reviewers for their hard work, time, and support that greatly helped them to select papers for this special issue.

\section{References}

1. Mitola J (2000) Cognitive radio: an integrated agent architecture for software defined radio. PhD dissertation, Royal Institute of Technology, Sweden, May

2. Haykin S (2005) Cognitive radio: brain-empowered wireless communications. IEEE J Select Areas Commun 23(5):201-220

3. First Report and Order in ET Docket no. 00-47, 16 FCC Red 17373 (2001) SDR report and order

4. FCC (2002) Spectrum policy task force report. ET Docket no. 02135, released November 7, pp 35-53

5. http://www.soumu.go.jp/main_sosiki/joho_tsusin/eng/presentation/ pdf/070713_1.pdf 